\title{
An Unusual Case of Unilateral Absent Infundibulopelvic Ligament in a Patient With Primary Infertility: Highlighting the Importance of Diagnostic Laparoscopy
}

\author{
Xin Yi Ho ${ }^{\mathrm{a}, \mathrm{b}}$, Chit Chong Khong ${ }^{\mathrm{a}}$
}

\begin{abstract}
During laparoscopy, pelvic anatomical variations may be expected but an absent infundibulopelvic ligament with present bilateral adnexa has not been reported in the literature thus far. We present a case report of a 33-year-old nulliparous lady who desires fertility and has had no previous surgery who underwent a laparoscopic cystectomy in which this was observed, highlighting the importance of diagnostic laparoscopy, and discuss the possible etiologies of how this may have arisen.
\end{abstract}

Keywords: Infundibulopelvic ligament; Primary infertility; Diagnosis; Absent; Diagnostic laparoscopy

\section{Introduction}

In the literature, there have been case reports of absent unilateral ovaries and fallopian tubes without any uterine anomalies [1-5]; however this in itself is rare. A comprehensive table of 17 case reports with patient characteristics has been summarized by Chen et al [4]. It is difficult to determine the incidence of adnexal malformations although a paper has suggested it to be one in 11,241 women [5]. There have been no reports of an absent infundibulopelvic ligament with an ipsilateral present and viable ovary. As we understand, in ovarian torsion when the ovarian vessels are occluded there is resultant pain and necrosis but this was not seen in our case. We thus present a case study and a discussion of the possible etiologies that have resulted in such a unique anatomy.

\section{Case Report}

A 33-year-old lady, married for 5 years, presented to our clinic

Manuscript submitted January 16, 2019, accepted February 11, 2019

aDepartment of Obstetrics \& Gynaecology, KK Women's and Children's Hospital, Singapore


ogy, KK Women's and Children's Hospital, 100 Bukit Timah Road, Singapore 229899, Singapore. Email: xinyi.ho@mohh.com.sg

doi: https://doi.org/10.14740/jmc3260 for primary infertility. She had been trying to conceive for 1 year. She has no past medical history or surgeries before. She was not on any long-term medication and has no drug allergies. Her periods were regular and she had no dysmenorrhea or dyschezia. There were no problems with coitus and no dyspareunia.

Upon investigation, it was found that her partner's semen analysis was normal. Her pelvic ultrasound showed a uterus size of $9.1 \times 6.2 \times 4.7 \mathrm{~cm}$ and endometrial thickness of 24 $\mathrm{mm}$. Within the endometrium was a $1.2 \mathrm{~cm}$ echogenic structure with a vascular stalk suggestive of an endometrial polyp. Multiple uterine fibroids were noted, of which the largest was a $4.5 \mathrm{~cm}$ subserosal fibroid at the lower left wall of the uterus. An anechoic cyst of $2.9 \mathrm{~cm}$ was noted in the right ovary and a corpus luteum in the left ovary.

She was thus counselled for a diagnostic laparoscopy, hydrotubation, hysteroscopy and polypectomy. Intra-operatively, a cystic mass was seen on the left side of the pelvis, with dense adhesions to omentum, anterior abdominal wall and bowel. It was difficult to tell if this was bowel, an ovarian cyst or a cystic degeneration of the left fibroid that was noted on the prior scan. A streak right ovary was seen and the right fallopian tube extended all the way to this mass. There was no spillage of dye from this right tube. The left fallopian tube was patent and spillage of dye was seen; however the left ovary was not seen.

The diagnostic laparoscopy was concluded and a pelvic magnetic resonance imaging (MRI) was arranged to delineate this cystic mass. It reported a well-circumscribed mildly lobulated mass lesion of $4.8 \times 3.5 \times 3.8 \mathrm{~cm}$ with fat and blood contents consistent with a dermoid cyst. As the right ovary was not seen, it was thought that this mass may represent either a left exophytic dermoid cyst or a right dermoid cyst which has gone over to the left. The left ovary was seen. There was a 2.9$\mathrm{cm}$ uniloculated cyst seen in the right adnexa with no ovarian tissue around it, possibly a paraovarian cyst. The left $4.2-\mathrm{cm}$ posterior wall subserosal fibroid was again noted.

The patient was counselled regarding the findings and was keen for a cystectomy. Histology of the endometrial curettings was benign endometrial polyps and early secretory endometrium. Her $\mathrm{Ca} 125$ was normal at 12.4. Importantly, she did not have any abdominal pain or recall having any significant episodes of pain prior.

In the second laparoscopy, adhesiolysis was done revealing a dermoid cyst containing pus and hair. There were plenty of vascular connections between this dermoid cyst and the omentum, anterior abdominal wall and bowel requiring dia- 




Figure 1. Omental adhesions with vascularity between anterior abdominal wall and dermoid cyst.



Figure 2. Dermoid cyst with pus and hair contents.

thermy (Figs. 1, 2). There was a right hydrosalpinx which appeared torted and elongated (Figs. 3, 4). Interestingly, there was no infundibulopelvic ligament found on the right side (Fig. 5). We managed to visualize the left tube and ovary after adhesiolysis (Fig. 6). The decision was made to perform the cystectomy, and detort and drain the right hydrosalpinx before the surgery was concluded. The final histology revealed a ma-

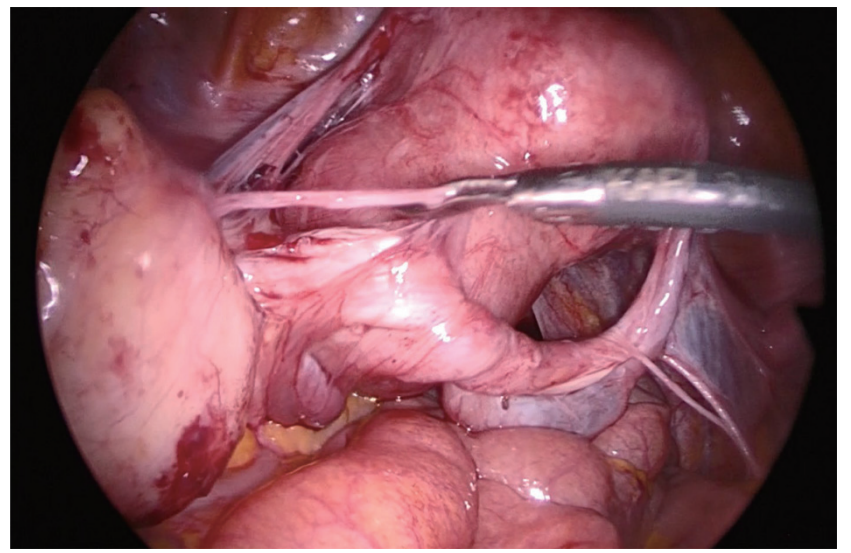

Figure 3. Right tube elongated and torted many times with hydrosalpinx noted.

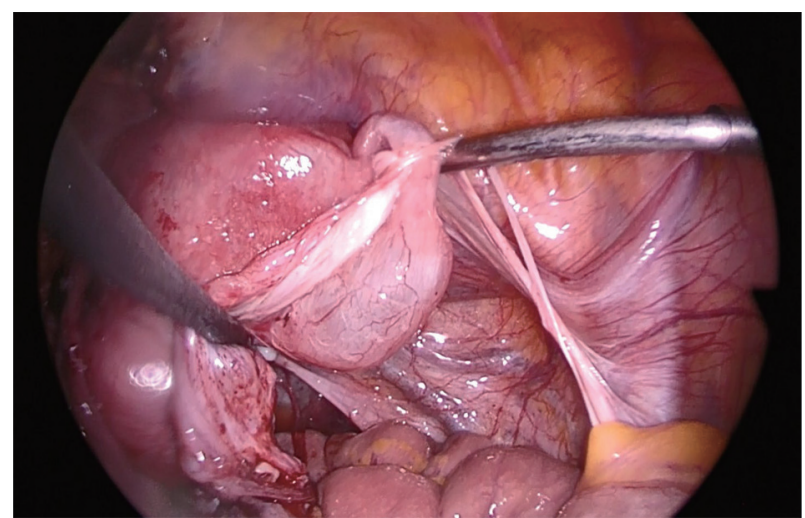

Figure 4. Hydrosalpinx noted and drained after detorsion completed.



Figure 5. Close up of absent infundibulopelvic ligament on the right side.

ture cystic teratoma with foci of infarction.

\section{Discussion}

During laparoscopy, pelvic anatomical variations may be expected [6]. However an absent infundibulopelvic ligament with present ipsilateral ovary is a very interesting discovery during a diagnostic laparoscopy because it defies our under-

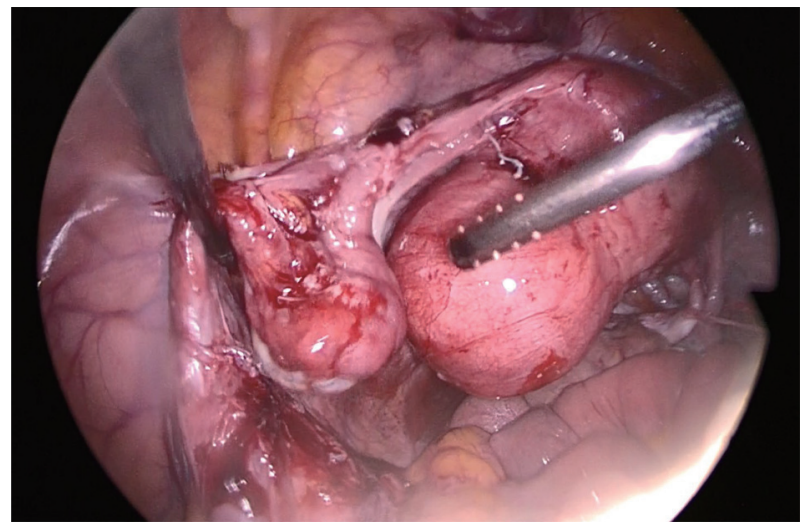

Figure 6. Left tube and ovary seen after adhesiolysis. 
standing of embryological development and vasculature.

The ovaries are derived from the gonadal ridge pinching off the mesonephros and descend to the gubernaculum in the women which lies at the fundus of the uterus [7]. The ovary is attached to the broad ligament by the mesovarium, to the uterus by the ovarian ligament, and to the pelvic side wall by the infundibulopelvic ligament or suspensory ligament of the ovary, which is the lateral margin of the broad ligament [7].

The ovaries are mainly supplied by the ovarian arteries which are direct branches of the abdominal aorta; however there are also anastomoses from the uterine artery (i.e. Sampson artery) [8].

We hypothesize that torsion may have occurred thus obliterating the infundibulopelvic ligament on the right side of our patient. This may also explain the elongated tube on the right. It is interesting that the patient had no symptoms and also necrosis did not occur. We postulate that this may be because the torsion had been a slow intermittent process and along the way collateral vasculature developed resulting in the supply of blood to the dermoid cyst via the uterine artery, omentum, bowels and anterior abdominal wall.

Our patient had unusual anatomy despite being asymptomatic. These findings demonstrate that diagnostic laparoscopy may be helpful in finding a cause and guiding treatment in asymptomatic patients with primary infertility.

\section{Grant Support}

Not applicable.

\section{Conflict of Interest}

The authors have no conflict of interest to declare.

\section{Ethics}

Written patient consent was obtained and Centralised Institutional Review Board approval was obtained prior to submission of this manuscript.

\section{References}

1. Eustace DL. Congenital absence of fallopian tube and ovary. Eur J Obstet Gynecol Reprod Biol. 1992;46(23):157-159.

2. Mylonas I, Hansch S, Markmann S, Bolz M, Friese K. Unilateral ovarian agenesis: report of three cases and review of the literature. Arch Gynecol Obstet. 2003;268(1):5760.

3. Elkington RR. Unexplained unilateral absence of fallopian tube and ovary. Internet $\mathrm{J}$ Gynecol Obstet. 2008;11(2).

4. Chen B, Yang C, Sahebally Z, Jin H. Unilateral ovarian and fallopian tube agenesis in an infertile patient with a normal uterus. Exp Ther Med. 2014;8(3):831-835.

5. Sivanesaratnam V. Unexplained unilateral absence of ovary and fallopian tube. Eur J Obstet Gynecol Reprod Biol. 1986;22(1-2):103-105.

6. Nezhat $\mathrm{CH}$, Nezhat F, Brill AI, Nezhat C. Normal variations of abdominal and pelvic anatomy evaluated at laparoscopy. Obstet Gynecol. 1999;94(2):238-242.

7. Beckmann C. Embryology and Anatomy, Obstetrics and Gynecology 5th edition, Lippincott Williams and Wilkins, Philadelphia, United States. 2006; p. 32-41.

8. Konar H. Development of genital organs and gonads. DC Dutta's Textbook of Gynecology 7th edition, Jaypee Brothers Medical Publishers, New Delhi, India. 2016; p. 31 . 